\title{
VASCULAR SEGMENTATION IN THREE-DIMENSIONAL ROTATIONAL ANGIOGRAPHY BASED ON MAXIMUM INTENSITY PROJECTIONS
}

\author{
Rui Gan*, Albert C. S. Chung*, Wilbur C. K. Wong* and Simon C. H. Yu \\ ${ }^{*}$ Dept. of Computer Science, the University of Science and Technology, HK. \\ ${ }^{\dagger}$ Dept. of Diagnostic Radiology and Organ Imaging, the Prince of Wales Hospital, HK.
}

\begin{abstract}
Three-dimensional rotational angiography (3D-RA) is a relatively new and promising technique for imaging blood vessels. In this paper, we propose a novel 3D-RA vascular segmentation algorithm, which is fully automatic and very computationally efficient, based on the maximum intensity projections (MIP) of 3D-RA images. Validation results on 13 clinical 3D-RA datasets reveal that, according to the agreement between the segmentation results and the ground truth, our method (a) outperforms both the Maximum a posteriori-expectation maximization (MAP-EM)-based method and the MAP-Markov random field (MAP-MRF)-based segmentation method, and (b) works comparably to the optimal global thresholding method. Experimental results also show that our method can successfully segment major vascular structures in 3D-RA and produce a lower false positive rate than that of the MAP-EMbased and MAP-MRF-based methods.
\end{abstract}

\section{INTRODUCTION}

Accurate description of the vascular tree is very important for clinical diagnosis and qualitative analysis. A three-dimensional (3D) vascular model generated by using automatic and semi-automatic segmentation techniques in angiograms can be very useful to clinicians. The 3D rotational angiography (3D-RA) is a relatively new imaging technique in neuroradiological interventions, and is a helpful intra- and inter-operative tool for assessing intracranial aneurysms and arteriovenous malformations [1] because it can provide $3 \mathrm{D}$ information of the cerebral vessels during treatment.

Using volume and surface rendering tools, which are mainly based on manual global thresholding, a time-consuming technique for searching the optimal threshold, 3D-RA vascular structures can be seen from any angle. However, due to the high noise level and the presence of reconstruction artifacts and unwanted non-vessel structures (e.g. bone), prior to visualization, preprocessing, such as noise reduction, is necessary. Meijering et al. proposed to use the edge-enhancing anisotropic diffusion (EED) [2], which takes both the contrast and orientation of edges into account, to reduce noise. They also demonstrated that, with respect to the noise reduction at the vessel walls and user-dependency of visualization, EED worked better than uniform filtering, Gaussian filtering, and regularized isotropic nonlinear diffusion. Nevertheless, EED can be costly in both time and memory. Besides segmentation by manual global thresholding for 3D-RA, Deschamps et al. introduced a level-set segmentation process for aneurysms in 3D-RA by using Fast-Marching [3]. The Gaussian distribution is adopted as the region descriptor for the inside as well as the outside of an aneurysm.

Statistically based parametric techniques have been widely used to classify vessels in magnetic resonance angiography (MRA), since they are efficient and easy to implement. Several researchers have demonstrated that, with a proper statistical mixture model for the observed intensity distribution of an angiogram, the expectation maximization (EM) algorithm followed by an estimator can be used to segment vascular structures $[4,5]$. However, to the best of our knowledge, this framework has not yet been applied to 3DRA.

In this paper, we present a novel, computationally efficient and fully automatic statistical segmentation technique for 3D-RA based on the maximum intensity projections (MIP). Our method takes an iterative approach, which is followed by a refinement technique, to segmenting a 3D vascular structure, progressively, based on segmentations of MIP images across iterations. During the experimental validation on 13 clinical 3D-RA datasets, manual segmentations authorized by a consultant radiologist were treated as the ground truth. Results showed that, according to the agreement between the segmentation results and the ground truth, our method (a) works better than the MAP-EM-based method as well as the MAP-MRF-based method (which is initialized by the MAP-EM-based method), and (b) works comparably to the optimal global thresholding method, in which the threshold was selected to give the segmentation with maximum agreement against the manual segmentation among all possible thresholds.

\section{PROBLEM STATEMENT}

Model selection is an important issue in the parametric statistical segmentation techniques. To the best of our knowledge, there is no published work relating to 3D-RA statistical modeling. According to the intensity range, three major classes can be found in a 3DRA dataset: background, non-vessel (e.g. bone and artifacts), and vessel (and aneurysm). Based on the observations in our previous work [6], we assume that each class has a Gaussian distribution of intensities. Furthermore, we found that, the means of the two classes in a relatively low intensity region were very close, however, their standard deviations were too large. This causes only one peak to be visible in the observed 3D-RA histogram. We have performed probing experiments on numerical phantom datasets, which simulated a 3D-RA image, to prove that the expectationmaximization (EM) estimation [7], which approximates the 3DRA volume intensity distribution with a triple-Gaussian mixture model, is quite unstable with respect to the parameter initialization of the non-vessel class. As such, we try to treat the background and the non-vessel classes as one class and approximate the intensity distribution of 3D-RA volume by a double-Gaussian mixture model. However, the segmentation error in the background was high due to the low threshold estimated by the maximum a posteriori (MAP) estimator [7]. After using the MAP-Markov random 
field (MAP-MRF)-based method [8] with the multi-label logistic (MLL) model [9] to refine the segmentations of the MAP-EMbased method, the noise ratios in the background were still very high. In the next section, we will present a new technique, which takes an iterative approach based on segmentations of MIP images across iterations, to segment 3D-RA vascular structures. The technique is a fully automatic algorithm with zero adjustable parameter; it is also simple, easy to implement, and very computationally efficient.

\section{MIP BASED STATISTICAL SEGMENTATION TECHNIQUE}

Our method is based on the fact that the maximum intensity projection (MIP) [10] operation can decrease the standard deviation of individual classes [11], which makes the modelling of the observed MIP intensity histogram (by a triple-Gaussian mixture model) more stable with respect to the parameter initialization [6].

\subsection{Iterative Segmentation Based on MIP Images}

The proposed segmentation algorithm utilizes an iterative approach. Given an intermediate segmentation at each iteration, an MIP image is generated from the $3 \mathrm{D}$ data and the corresponding $3 \mathrm{D}$ position of each pixel in the MIP image is recorded. The MAP-EMbased algorithm with the triple-Gaussian mixture model, which is given by (assume that $\mu_{1}<\mu_{2}<\mu_{3}$ and $\sum_{k=1}^{3} \omega_{k}=1$ ),

$$
p(x)=\sum_{k=1}^{3} \omega_{k} \frac{1}{\sqrt{2 \pi \sigma_{k}^{2}}} \exp \left[-\frac{1}{2}\left(\frac{x-\mu_{k}}{\sigma_{k}}\right)^{2}\right]
$$

is used to segment the MIP image into vessel (modeled by the Gaussian distribution with the largest mean) and background (modeled by the joint distribution of the other two Gaussians). The threshold, $t$, is obtained by the MAP estimator. Providing that not all the pixels are classified as background, voxels of 3D-RA, whose corresponding pixels in the segmented MIP image are classified as vessel, are added to the vessel class; the algorithm, therefore, proceeds to the next iteration. At any successive iterations, voxels, which are classified as vessel, are excluded in the MIP computation. The termination of the iterative procedure is determined by a mechanism based on the sum of absolute differences (SAD). To avoid unnecessary intensity interpolation, we set the projection direction to one of the three principle axes.

At successive iterations, the number of vessel pixels in the MIP images decreases as more vessel voxels are excluded in the MIP computation. Thus the MIP image intensity distribution may evolve and the estimation of the background class may end with overfitting, which implies that the background distribution is not modeled by the first two Gaussians exclusively, instead, is modeled by the overall triple-Gaussian distribution. As shown in Fig. 1 , overfitting results in a low threshold and causes a high segmentation error in the background. Therefore, once we detect overfitting, the iterative approach should be terminated.

We propose a mechanism, which is based on SAD, to detect overfitting in the background class. At the $k^{t h}(k>0)$ iteration, without any prior knowledge, we estimate the background distribution of the current MIP image, $h_{k}^{b}$, from the threshold calculated at the previous iteration, $t^{k-1}$. Thus, for intensity level $i, h_{k}^{b}(i)$, is

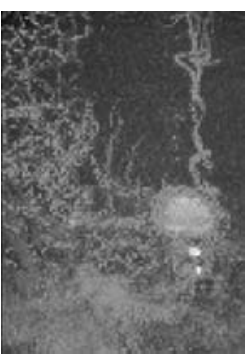

(a)

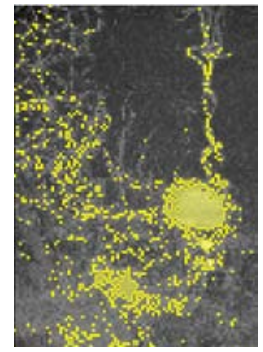

(b)

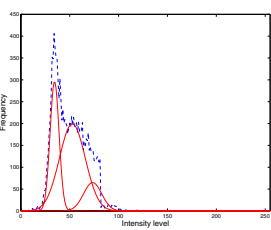

(c)
Fig. 1. (a) The MIP image from the last iteration. (b) The segmented MIP image. (c)The intensity histogram (dashed line) along with the estimated mixture model.

defined by,

$$
h_{k}^{b}(i)=\left\{\begin{array}{ll}
h_{k}(i) & \text { if } i<t^{k-1} \\
0 & \text { otherwise }
\end{array},\right.
$$

where $h_{k}$ is the observed MIP image histogram at the current iteration. After the EM algorithm, let $h_{k}^{1}$ be the joint distribution of the two Gaussians in a relatively low intensity region and $h_{k}^{2}$ be the overall triple-Gaussian distribution. We then calculate two SAD values: $S A D\left(h_{k}^{b}, h_{k}^{1}\right)$, between $h_{k}^{b}$ and $h_{k}^{1}$, and $S A D\left(h_{k}^{b}, h_{k}^{2}\right)$, between $h_{k}^{b}$ and $h_{k}^{2}$. With these two values, the overfitting problem can be avoided as follows: (a) if $S A D\left(h_{k}^{b}, h_{k}^{2}\right) \leq S A D\left(h_{k}^{b}, h_{k}^{1}\right)$, i.e., the overall triple-Gaussian distribution models the background class, then the iterative procedure should be terminated; or (b) otherwise, continues. Let $K$ be the iteration at which we detect overfitting. In order to segment all vessel voxels out, the threshold from the previous iteration, $t^{K-1}$, is employed as a global threshold to segment the current 3D-RA volume. This is equivalent to using $t^{K-1}$ to globally segment MIP images successively, if we keep on iterating, until no vessel voxel is found. The iterative segmentation algorithm is outlined in Algorithm 1.

Algorithm 1 The iterative segmentation algorithm based on MIP.

Initialization: A 3D-RA volume, $V$, and an empty volume, $X$, which is of the same size as $V$.

Step 1. Create an MIP image from $V$, while record the 3D position of each pixel in the MIP image. Estimate the parameters in the triple-Gaussian mixture model using the EM algorithm.

Step 2. Calculate $S A D\left(h^{b}, h^{1}\right)$ and $S A D\left(h^{b}, h^{2}\right)$. If $S A D\left(h^{b}, h^{2}\right) \leq S A D\left(h^{b}, h^{1}\right)$, goto Step 4 .

Step 3. Segment the MIP image by the MAP estimator. Classify the voxels in $X$, whose corresponding pixels are classified as vessel, to vessel, and set their intensity values in $V$ to null. Goto Step 1.

Step 4. Use the threshold from the previous iteration to globally segment $V$, and update the voxel classification in $X$. Return $X$ as the final 3D segmentation.

\subsection{MIP-based Segmentation in Multiple Projections}

In order to exploit more information in 3D-RA, we extend the Algorithm 1 in multiple projections. Let $\mathcal{D}=\left\{d_{1}, d_{2}, \cdots, d_{n}\right\}$ be 
a set of directions. (In our experiments, in order to avoid timeconsuming intensity interpolation, we set $\mathcal{D}$ to the three principle axial directions.) Along each direction $d(d \in \mathcal{D})$, we apply the Algorithm 1 (i.e. MIP images are computed along $d$ ) and get a segmentation, $X_{d}$. Then we obtain a set of segmentations, $\mathcal{X}_{\mathcal{D}}=\left\{X_{d_{1}}, X_{d_{2}}, \cdots, X_{d_{n}}\right\}$. Given a 3D neighborhood system $\mathcal{N}$, the final segmentation, $X^{*}$, is obtained from $\mathcal{X}_{\mathcal{D}}$ by employing the iterated conditional modes (ICM) [12] to minimize an energy function, which embodies both the knowledge of the observed image and spatially smooth. Given a segmentation $X$, the energy, $E(X)$, is defined as,

$$
E(X)=\sum_{v \in V}\left(L\left(X_{v} \mid I_{v}\right)+\lambda \sum_{v^{\prime} \in \mathcal{N}(v)} P\left(X_{v}, X_{v^{\prime}}\right)\right),
$$

where $X_{v}$ is the classification (vessel or background) of a voxel, $v$, in $X$, and $I_{v}$ is the observed intensity value of $v$. We define

$$
L(x \mid i)=\frac{1}{\left|\mathcal{X}_{\mathcal{D}}\right|} \sum_{X \in \mathcal{X}_{\mathcal{D}}} L_{X}(x \mid i)
$$

and

$$
P\left(X_{v}, X_{v^{\prime}}\right)=\left\{\begin{array}{ll}
-1 & X_{v}=X_{v^{\prime}} \\
0 & X_{v} \neq X_{v^{\prime}}
\end{array},\right.
$$

where $\left|\mathcal{X}_{\mathcal{D}}\right|$ is the size of $\mathcal{X}_{\mathcal{D}}$ and $L_{X}(x \mid i)$ is the negative loglikelihood energy of classification $x$ to intensity value $i$ in segmentation $X$, which can be estimated via histograming the intensity values for individual classes in $X_{d}$. The constant $\lambda$ in Eqn. 3 controls the degree of spatial smoothness and is set to 1 in our implementation.

\section{Summary of the MIP-based Segmentation Algorithm:}

Step 1. Along the three principle axial directions, separately exploit the Algorithm 1, and obtain three segmentations.

Step 2. Use ICM to compute the final segmentation by minimizing Eqn. 3.

\section{VALIDATION AND EXPERIMENTAL RESULTS}

To obtain a quantitative assessment of our method, we manually segmented 13 clinical 3D-RA datasets and the segmentations were modified and verified by a consultant radiologist with 16 years experience at our collaborating hospital. These manual segmentations were treated as the ground truth of the 13 datasets. A segmentation was then evaluated against the manual segmentation with three different statistical measures: (1) Dice similarity coefficient (DSC) [13]; (2) True positive rate (TPR); and (3) False positive rate (FPR). TPR measures the sensitivity and FPR measures the specificity, while, DSC measures the agreement between two segmentations since it takes both sensitivity and specificity into account. As such, DSC can be treated as a more objective measure in our validation.

For comparing the results, we applied the MAP-EM-based method with a double-Gaussian distribution (referred as MAPEM), and the MAP-MRF-based method with the multi-label logistic (MLL) model (referred as MAP-MRF), which refined the segmentations of MAP-EM, for the 13 datasets. Then, we applied the optimal global thresholding method (refereed as OGT), in which the threshold was selected to give the segmentation with

\begin{tabular}{|l|c|c|c||c|c|}
\hline Method & DSC & TPR & FPR & Mean & SD \\
\hline MIP-MP & 0.8937 & 0.8822 & 0.0006 & 0.0038 & 0.0193 \\
\hline MIP & 0.8510 & 0.8332 & 0.0009 & 0.0321 & 0.0302 \\
\hline MAP-MRF & 0.5941 & 0.9862 & 0.0093 & 0.3187 & 0.2096 \\
\hline MAP-EM & 0.3638 & 0.9993 & 0.0245 & 0.4873 & 0.1912 \\
\hline OGT & 0.8670 & 0.8406 & 0.0007 & - & - \\
\hline
\end{tabular}

Table 1. Columns "DSC", "TPR" and "FPR" give evaluation results of different segmentation methods on a 3D-RA dataset against the authorized manual segmentation. Columns "Mean" and "SD" show the means and the standard deviations of the relative differences between the DSCs of OGT and the DSCs of other methods on 13 datasets.

maximum DSC against the manual segmentation among all possible thresholds. The MIP-based segmentation algorithm along one projection (Algorithm 1) (referred as MIP) was also tested. The whole approach of our method (along multiple directions) is referred as MIP-MP. In total, there are 5 different methods as listed in Table 4.

In the table, columns "DSC", "TPR" and "FPR" present the evaluation results on one 3D-RA dataset. Columns "Mean" and "SD" show the means and the standard deviations of the relative differences between the DSCs of OGT and the DSCs of the other methods on the 13 datasets. According to the values in columns "DSC" and "Mean", the evaluation results showed that MIP-MP worked better (larger DSC) than MAP-EM and MAP-MRF on all 13 datasets. (The mean and the standard deviation of MIP-MP were 0.0038 and 0.0193 . Meanwhile, for MAP-EM, their values were 0.4873 and 0.1912 , and for MAP-MRF, their values were 0.3187 and 0.2096.) Moreover, it is noted that, according to DSC values, MIP-MP outperformed OGT on 6 datasets and worked comparably to OGT on another 7 datasets.

We also found that the FPRs of the segmentations obtained by MIP-MP were consistently smaller than by MAP-EM and MAPMRF, and comparable to by OGT. (Column "FPR" in the table shows the typical values of one dataset.) In addition, MIP-MP consistently produced larger TPRs than that of MIP on all 13 datasets thanks to the combination of segmentations from multiple directions (Section 3.2). It is also noticeable that MAP-EM and MAPMRF produced high FPRs as well as high TPRs on all 13 datasets.

The processing time of MIP-MP, MAP-EM and MAP-MRF were, on average, around 8,2 and 30 seconds respectively, for segmenting a $3 \mathrm{D}-\mathrm{RA}$ of size $100 \times 100 \times 100$ on a $2.26 \mathrm{GHz} \mathrm{PC}$. Meanwhile, to manually find a good global threshold, popular in 3D-RA processing, is always time consuming.

Fig. 2 shows six ROIs, which are segmented by (a) a consultant radiologist, (b) MAP-EM, (c) MAP-MRF, (d) MIP, (e) MIPMP, and (f) OGT. To visualize segmentations, the volume rendered images were created using the Visualization Toolkit (VTK). Fig. 3 shows (a) the axial MIP image, three volume rendered images based on segmentations by (b) a radiologist, (c) MAP-MRF, and (d) MIP-MP. Results revealed that, MIP-MP has successfully segmented the major vascular structures in 3D-RA, and it produced less noise than MAP-MRF.

\section{CONCLUSIONS AND DISCUSSION}

The EM-based segmentation method, because of its simplicity and computational efficiency, has been widely used for image segmen- 


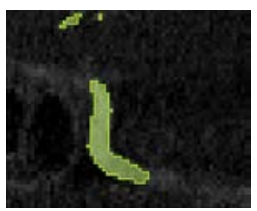

(a) Manual

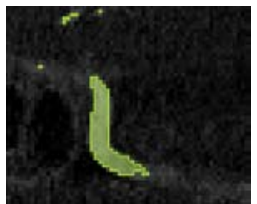

(d) MIP

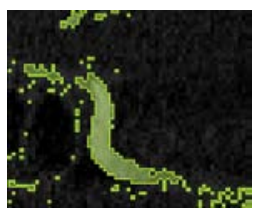

(b) MAP-EM

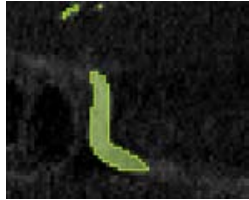

(e) MIP-MP

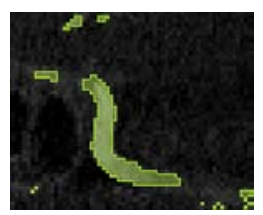

(c) MAP-MRF

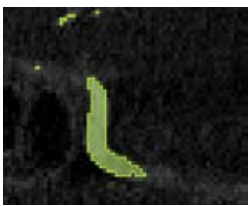

(f) OGT
Fig. 2. ROIs segmented by different methods. ("MIP" and "MIPMP" are the Algorithm 1 and the whole approach of our algorithm.)

tation. Based on our experimental results on 13 clinical 3D-RA datasets, it is shown that, the double-Gaussian mixture model gives unsatisfactory segmentations because the estimated global thresholds are too low which give high false positive rates (FPRs) in the segmentations. Even if with the refinement by the MRF-based method, the problem cannot be alleviated by much. In this paper, we have proposed a novel 3D-RA vascular segmentation technique based on the iterative segmentations of maximum intensity projections (MIP) along multiple projections. In an MIP image, the variances of each structural class (background, non-vessel and vessel) become smaller. This makes the modelling of the observed MIP intensity histogram more stable. For validation, we compared our method with (a) the MAP-EM-based method, (b) the MAP-MRF-based method and (c) the optimal global thresholding method (based on manual segmentations obtained with the help of a radiologist). Validation results on 13 3D-RA datasets showed that our method consistently gave higher Disc similarity coefficients (DSCs) than the MAP-EM-based and the MAP-MRF-based methods, and gave comparable DSCs to the optimal global thresholding method. Experimental results on 13 datasets revealed that our method can successfully segment the major vascular structures and produce less noise than the MAP-EM-based and the MAPMRF-based methods. Moreover, the computational efficiency of our method is high (on average, around 8 seconds per dataset of $100 \times 100 \times 100$ voxels). Our future work is to apply and extend our method to other medical angiograms, for example, PC-MRA and TOF-MRA.

\section{REFERENCES}

[1] J. Moret et al., "3d rotational angiography: Clinical value in endovascular treatment," Medicamundi, vol. 42, pp. 8-14, 1998

[2] E. H. W. Meijering et al.,"Evaluation of diffusion techniques for improved vessel visualization and quantification in threedimensional rotational angiography," in MICCAI, 2001, pp. $177-185$.

[3] T. Deschamps, Curve and Shape Extraction with Minimal Path and Level-Sets techniques. Applications to $3 \mathrm{D}$ Medical Imaging, Ph.D. thesis, Philips Research Laboratories Paris/LEP, 2001.

[4] A. C. S. Chung, Vessel and Aneurysm Reconstruction using

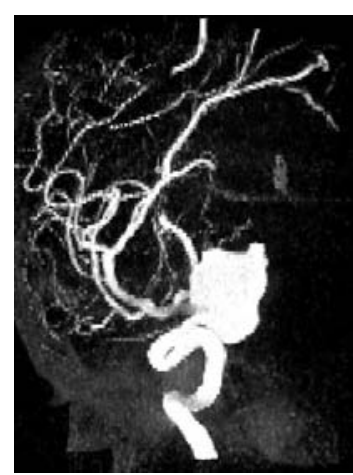

(a) Axial MIP

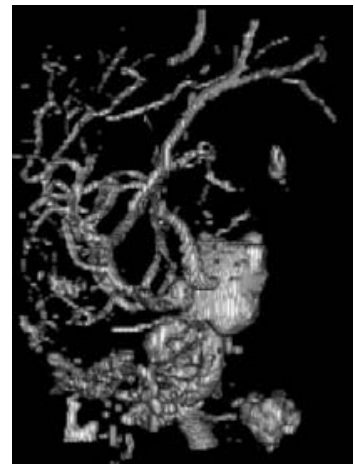

(c) MAP-MRF

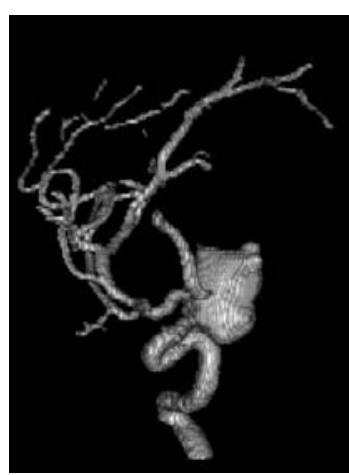

(b) Manual

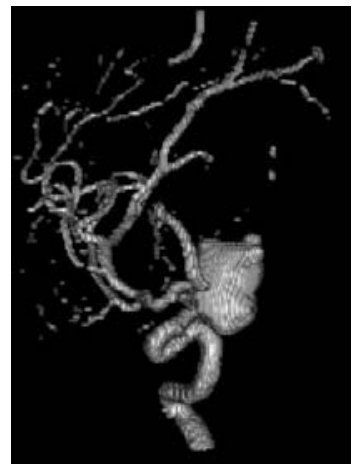

(d) MIP-MP
Fig. 3. Volume rendered images based on different methods.

Speed and Flow Coherence Information in Phase Contrast Magnetic Resonance Angiograms, Ph.D. thesis, University of Oxford, 2001.

[5] D. L. Wilson et al., "An adaptive segmentation algorithm for time-of-flight mra data," IEEE TMI, vol. 18, no. 10, pp. 938945, 1999.

[6] R. Gan et al., "Statistical vascular segmentation in threedimensional rotational angiography based on maximum intensity projections," submitted to ECCV 2004.

[7] C. Bishop, Neural Networks for Pattern Recognition, Clarendon Press, 1995.

[8] S. Geman et al., "Stochastic relaxation, gibbs distributions, and the bayesian restoration of images," IEEE PAMI, vol. PAMI-6, no. 6, pp. 721-741, 1984.

[9] S. Z. Li, Markov random field modeling in computer vision, Springer-Verlag, 1995.

[10] S. Rossnick et al., "Three dimensional display of blood vessels in mri," Boston, 1986, IEEE Computer in Cardiology, pp. 193-196.

[11] S. Schreiner et al., "The importance of ray pathlengths when measuring objects in maximum intensity projection images," IEEE TMI, vol. 15, no. 4, pp. 568-579, 1996.

[12] J. Besag, "On the statistical analysis of dirty pictures," Journal of the Royal Statistical Society. Series B (Methodological), vol. 48, no. 3, pp. 259-302, 1986.

[13] A. P. Zijdenbos et al., "Morphometric analysis of white matter lesions in mr images: method and validation," IEEE TMI, vol. 13, no. 4, pp. 716-724, 1994. 